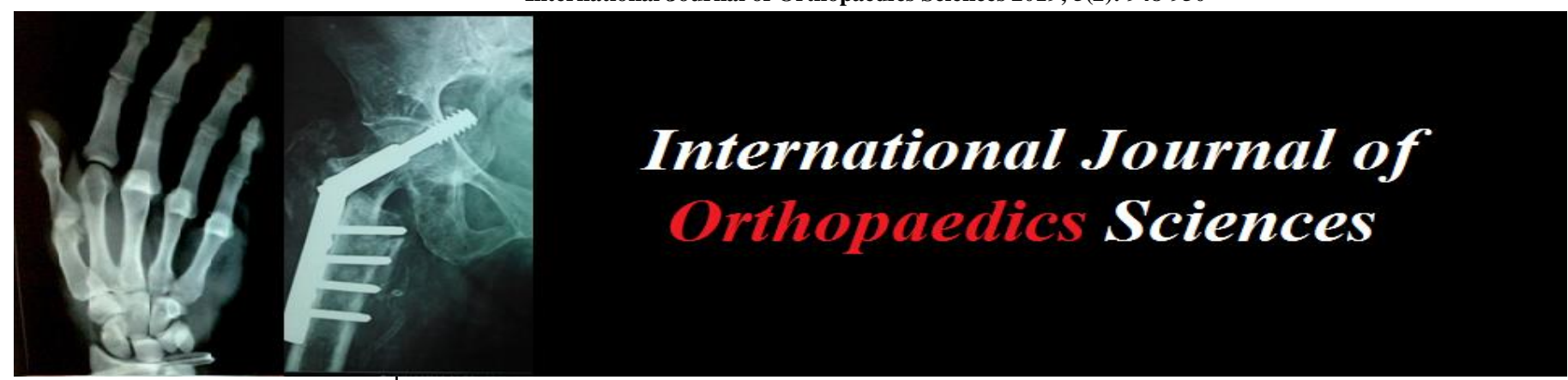

ISSN: $2395-1958$

IJOS 2019; 5(2): 948-950

(C) 2019 IJOS

www.orthopaper.com

Received: 21-02-2019

Accepted: 23-03-2019

Dr. Ruchit Shah

Eras Medical College, Lucknow,

Uttar Pradesh, India

Dr. Urvi Khambhati

Department of Radiodiagnosis,

Sir T Hospital, Bhavnagar,

Gujarat, India

\section{A rare case report of osteochondroma on dorsal surface of scapula}

\section{Dr. Ruchit Shah and Dr. Urvi Khambhati}

DOI: https://doi.org/10.22271/ortho.2019.v5.i2n.1461

\begin{abstract}
Osteochondroma is one of the most common cartilage-capped outgrowth and it grows underneath the periosteum through ossification of the cartilage. Most common age of presentation is noted in second decade of life near long bones of knee joint which are distal end of femur and proximal end of tibia. Osteochondroma as seen radiologically are of two types: sessile and pedunculated seen as outgrowth from scapula. Occurrence of osteochondroma in scapula and in $1^{\text {st }}$ decade of life is very rare It mainly causes cosmetic disfigurement and pain due to fracture, malignant transformation or pseudowinging or compression of neural or vascular structures. Here presenting a rare case report of osteochondroma on dorsal surface of scapula treated with complete surgical excision and functional improvement of the patient.
\end{abstract}

Keywords: Osteochondroma of scapula, benign bone tumor

\section{Introduction}

Osteochondroma is benign neoplasm characterised by overgrowth of cartilage and bone that occurs at the end of the bone near the growth plate and grows beneath periosteum through ossification of the cartilage. It most often occurs between ages 10 and 30 affecting males and females equally ${ }^{[6]}$. Most often it affects the metaphysis of long bones around the knee joint like distal femur and proximal tibia or may involve proximal shaft of humerus. Occurrence of osteochondroma in other bones is rarely reported ${ }^{[7]}$. About 1-3\% of cases of osteochondroma are reported in scapula ${ }^{[6]}$. Generally osteochondroma is asymptomatic but as it enlarges in size it causes symptoms due to mechanical pressure, neuro vascular impingement, or bursa formation, fracture of the bony stalk, cosmetic disfigurement or rarely malignant transformation of the cartilage cap. Hereby presenting a rare case of single, atypical large osteochondroma on scapula associated with symptoms of mechanical discomfort and cosmetic disfigurement in a 10 year old boy which was diagnosed with xrays and treated with complete surgical excision and thereby palliation of the symptoms.

\section{Presentation of the case}

A 10 years old male child presenting with chief complaints of discomfort during sleeping in supine position and swelling which was gradually increasing in size from that of a pea to size of a tennis ball since 3 years. There was no associated history of trauma, pain, fever or weight loss. Symptoms were gradually progressive with no similar family history. Informed consent was taken from the patient. Clinically on inspection there was a well-defined approximately $4 \times 2 \mathrm{cms}$ solitary oval swelling on the dorsal surface of left scapula, about 4-5 cms away from medial border of scapula. It was located near centre of infraspinous fossa. The skin over the swelling was normal, mobile, not adherent to the swelling. There was no associated tenderness, redness or local increase in temperature or other signs of inflammation. The consistency of the lesion was hard with well-defined margins. It was non mobile, nontranslucent. Swelling was fixed to scapula which suggested its origin from the bone. There was no discomfort in movement of the shoulder or numbness, paresthesia, weakness or pain in left upper limb. 


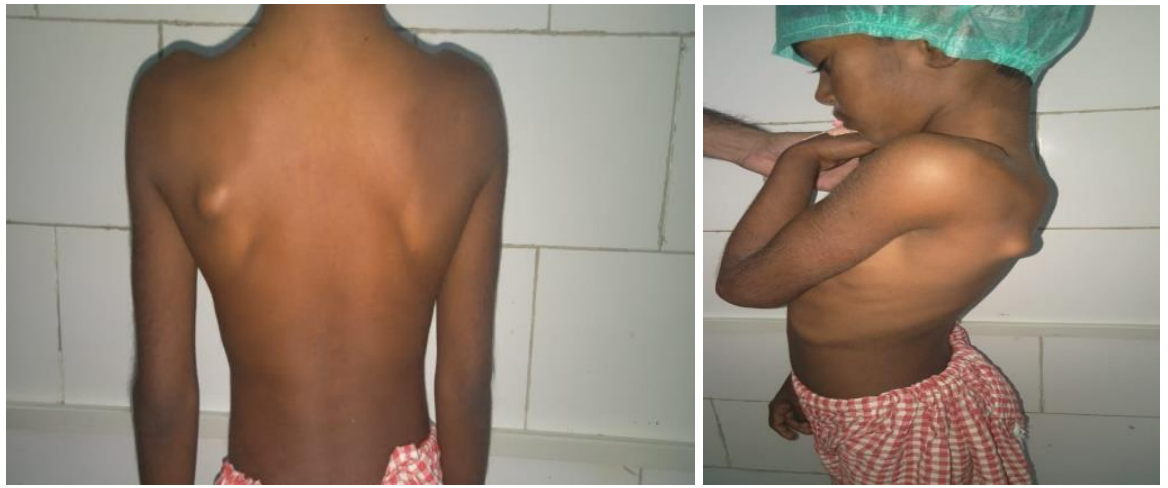

Fig (A) \& (B): Clinical presentation of the lesion from back from lateral view
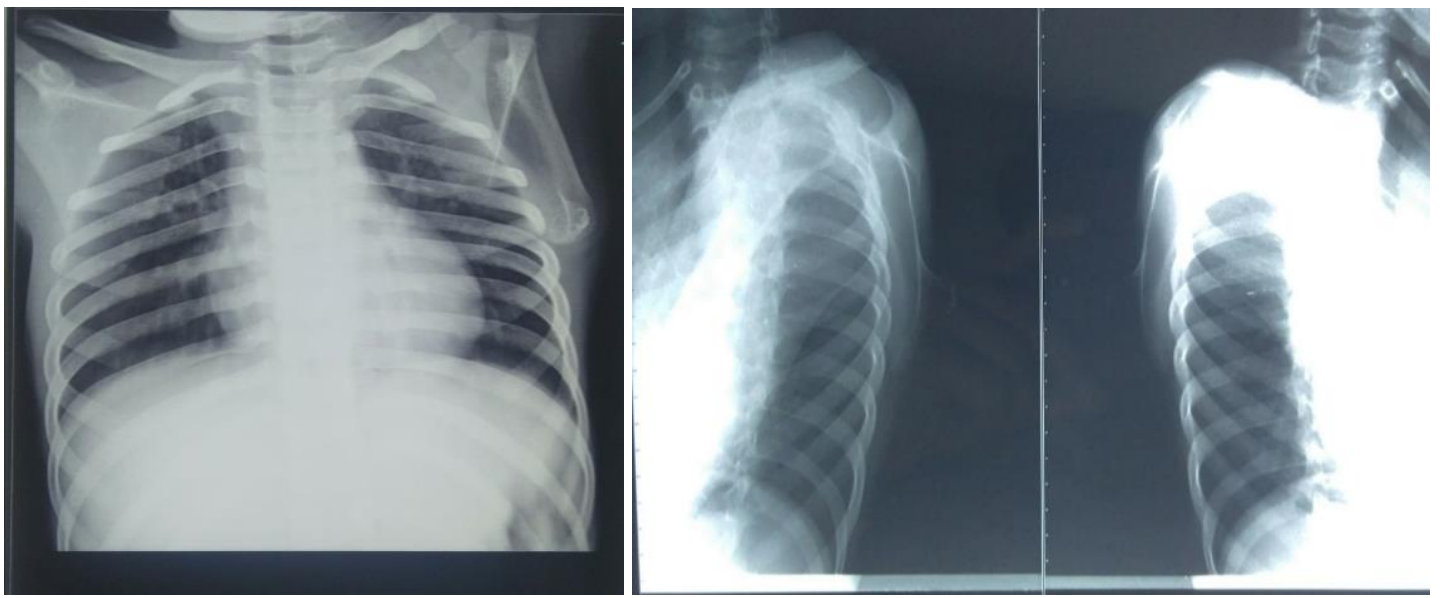

Fig (C) \& (D): Chest X-Ray PA and lateral views showed single well-defined sessile swelling with arising from dorsal surface of the left scapula with cartilaginous cap covering it.

Due to typical features of single sessile swelling with cartilage cap, provisional diagnosis of osteochondroma was made from xrays and further poor socioeconomic condition of the patient did not permit for further evaluation of lesion. Further, as a part of management of the patient, he was subjected to excisional biopsy under general anesthesia. After admission for the surgery, the patient was positioned in the lateral decubitus position with slight abduction and angulation forwarded with axillary roll. The skin overlying the lesion was prepared with antiseptics and Curvilinear incision was given on the swelling and exposed by dissection of overlying muscle and fascia. The lesion was excised in total.

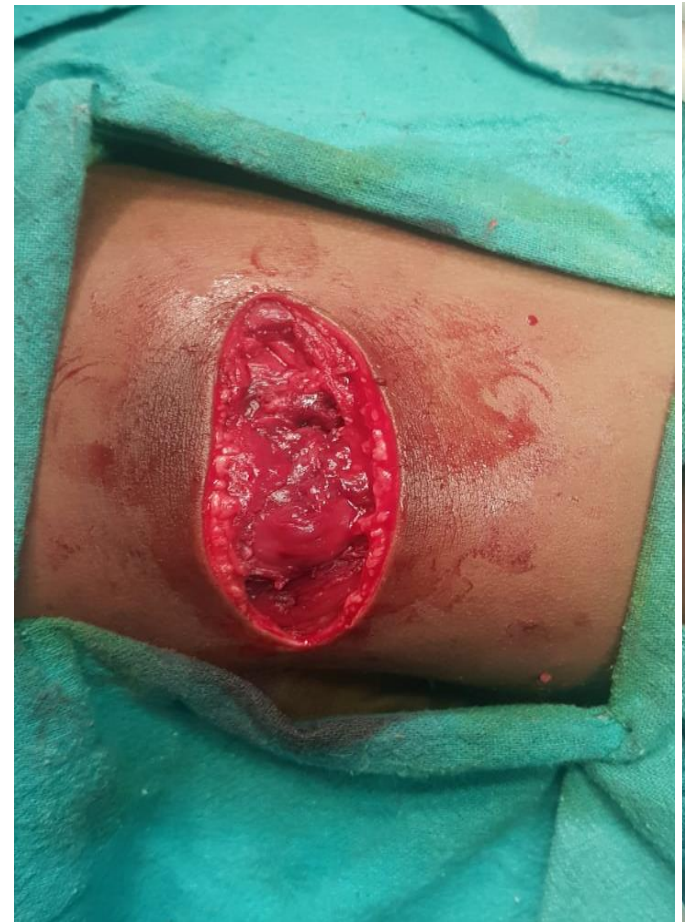

Fig E: skin and soft tissue incision exposing the lesion.

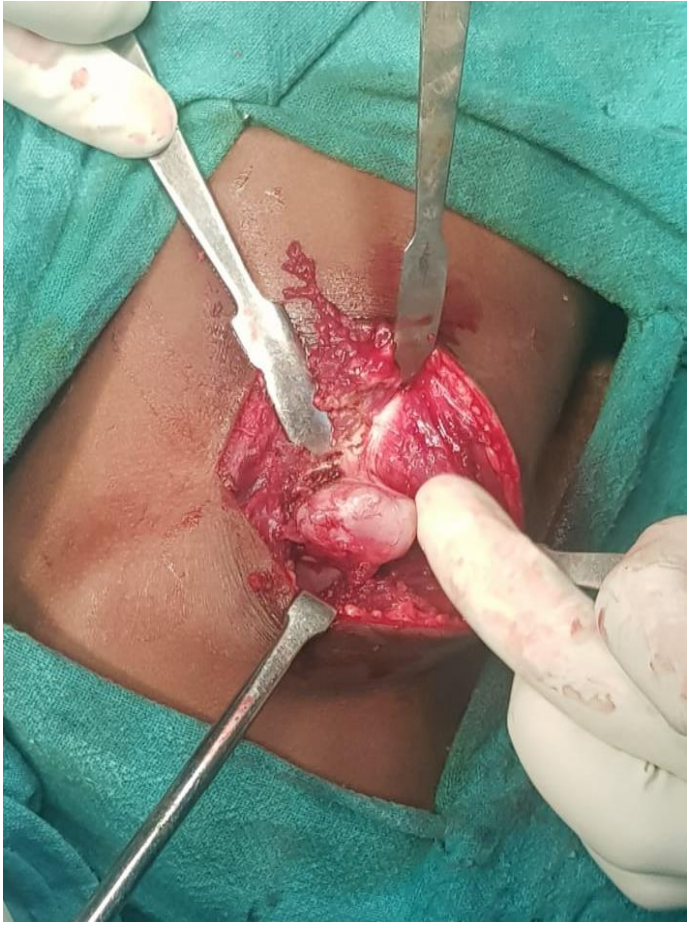

Fig F: swelling with cartilage cap seen. 


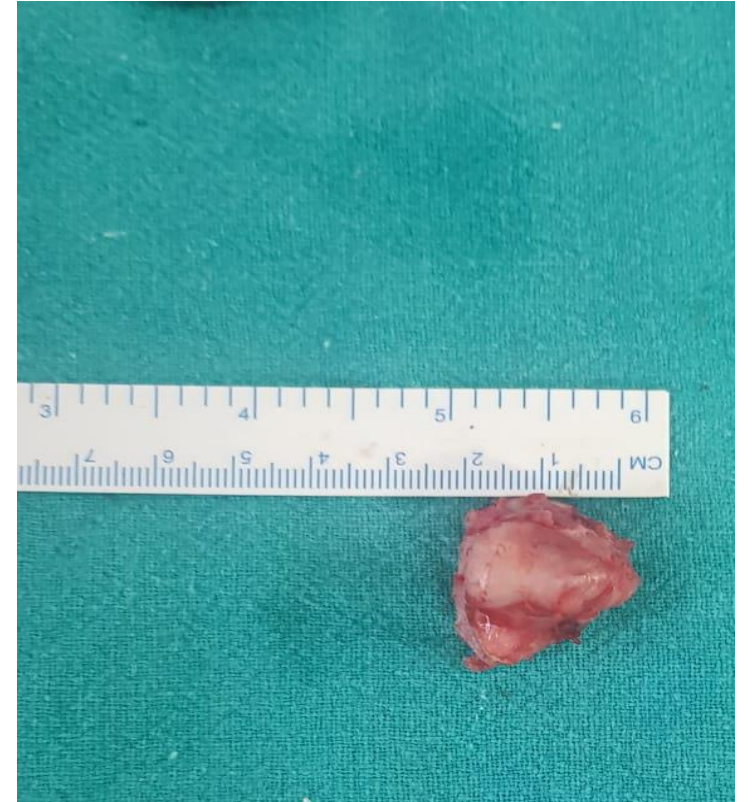

Fig G: specimen showing the morphology and approximate size of the lesion.

Physical examination showed the swelling was of whitish grey in color, irregularly lobulated in shape and hard in consistency. The size and weight of the swelling were $4 \times 2 \times 1$ and $25 \mathrm{gm}$ respectively. Cut surface of the swelling was greyish white in color with a cartilage cap covering it. Histopathology confirmed the diagnosis of osteochondroma. Postoperatively there was total relief of symptoms with no complications and no evidence of recurrence at follow up period of 6 months and 1 year.

\section{Discussion}

Osteochondroma is one of the most common benign neoplasm characterised by outgrowth of cartilage and bone that occurs at the end of the bone near the growth plate and grows beneath periosteum through ossification of the cartilage ${ }^{[6]}$. According to the present literature about $14 \%$ of OC are due to an autosomal-dominant disorder characterised by multiple OC and $86 \%$ are solitary OC without genetic preponderence ${ }^{[1,3]}$. In about in $1-2 \%$ of individuals, Solitary OC is seen ${ }^{[4]}$. With growth of skeleton, an osteochondroma may grow larger but after skeletal maturity has been reached, the osteochondroma stops growing. Most common sites of occurrence are the metaphysis of distal femur, proximal tibia and proximal humerus.

Early diagnosis of this benign tumor is a challenging task as in majority of the cases it is asymptomatic or there may be some amount of cosmetic disfigurement or slight limitation of joint movement. Also the rate of growth of these tumors is very less and so they are incidentally diagnosed in majority of the cases. However, rarely the tumors may grow bigger to cause compression of the surrounding neuro vascular structures causing pain, numbness or wining of scapula, or due to trauma there may be pain secondary to fracture in the stalk or in very rare and long standing cases there may be malignant transformation from the cartilaginous cap $\left.{ }^{[2,} 3\right]$. Incidence of osteochondroma in flat bones like scapula is reported to be around $4 \%{ }^{[4,5,10]}$. In our case, the patient had mechanical distress suring sleeping in supine position with some amount of cosmetic disfigurement but no complaint of pain. Collaborating the clinical findings along with the radiological findings by $\mathrm{x}$-rays suggested the diagnosis of exostosis and osteochondroma and it was excised completely which was confirmed by histopathological report. The patient had complete resolution of symptoms and on follow up studies at 6 and 12 months there was no evidence of recurrence.

\section{Conclusion}

We have hereby presented a case report of osteochondroma on dorsal surface of scapula associated with cosmetic disfigurement and mechanical irritation diagnosed radio graphically by $\mathrm{x}$-rays and was excised in total and thereby relief in symptoms. There was no recurrence on follow up studies. So by early identification and diagnosis of this benign entity we aim to alleviate the patients symptoms completely even before complications or malignant transformation occurs.

\section{References}

1. Unni KK, Inwards CY, Dahlin's Bone Tumors. General Aspects and Data on 10,165 Cases, Wolters Kluwer/Lippincott Williams \& Wilkins, Philadelphia, PA, USA, 6th edition, 2010.

2. Bovee JVMG, Heymann D, Wuyts W, Osteochondroma. in WHO Classification of Tumours of Soft Tissue and Bone, C. D. M. Fletcher, J. A. Bridge, P. C. W. Hogendoorn, and F. Mertens, Eds., International Agency for Research on Cancer, Lyon, France, 2013, 250-251.

3. Murphey MD, Choi JJ, Kransdorf MJ, Flemming DJ, Gannon FH. Imaging of osteochondroma: variants and complications with radiologic-pathologic correlation, Radiographics. 2000; 20(5):1407-1434. 